\title{
Study on the Correlation between Polysaccharide Gel and Spleen Qi Deficiency Symptoms
}

\author{
Bill Che ${ }^{1}$, Linan $\mathrm{Li}^{1}$, Jiarui $\mathrm{Li}^{2,3}$, Li Li ${ }^{2,3}$, Hong Meng ${ }^{2,3}, \mathrm{Jim} \mathrm{Liu}^{1^{*}}$ \\ ${ }^{1}$ Department of Research and Development Daily Chemicals Cosmetology, Infinitus Co. Ltd., Guangzhou, China \\ ${ }^{2}$ Department of Cosmetics, Beijing Technology and Business University, Beijing, China \\ ${ }^{3}$ Beijing Key Laboratory of Plants Resource Research and Development, Beijing, China
}

\section{*Corresponding author: Jim Liu, Department of Research and Development Daily Chemicals Cosmetology, Infinitus Co. Ltd., 17th floor, Infinity center, 12 Zhujiang west road, Zhujiang new town, Tianhe district, Guangzhou 510000, China Tel.: +86203816 8888 \\ Email: Jim.Liu@infinitus-int.com}

Received February 25, 2020

Revised March 03, 2020

Accepted March 25, 2020

Published March 30, 2020

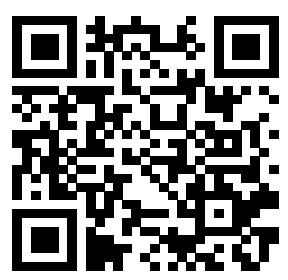

\begin{abstract}
Purpose: It is verified that polysaccharide gel can improve the spleen qi deficiency, especially the skin phenomenon caused by spleen qi deficiency. Methods: A mouse model of spleen qi deficiency syndrome was established by eating disorder. Four kinds of polysaccharide gels were applied to the skin of the backs of mice, and their weights, spleen-thymus index, skin sections, and skin indexes were used for evaluation. Results: The spleen qi deficiency mice had lower water content, lower gloss, and increased oil secretion, which were significantly different from those of the control group $(p<0.01)$. The polysaccharide gel acts on mice with spleen qi deficiency, compared with the model group, the skin moisture content is increased, the gloss is increased, and the oil secretion is reduced, and it is significant. Conclusion: The polysaccharide gel can improve the symptoms of spleen qi deficiency, especially the dry and dull skin caused by spleen qi deficiency, and provide a basis for the subsequent application of polysaccharides in cosmetics to improve the skin symptoms of spleen qi deficiency.
\end{abstract}

Keywords: Spleen Qi deficiency, Skin, Dendrobium polysaccharides, Saussurea Ivolucrata polysaccharides, Tremella polysaccharides

\section{Introduction}

中医学认为 “有诸内必形诸于外”。《黄帝内经》曰: “人 之气血精神也, 所以奉生而周于性命者也。”气血是人体的基 本物质, 代表人体的正气, 是机体内部平衡的基本因素。气血关 系到五脏、经络、四肢百骸, 气血的平衡, 定会表现于面部 皮肤 (Qiu，2018)。张仲景也认为, 人体肤色红润有光泽, 肌 肉坚实有弹性及毛发润泽与否等外在表现与营卫气血的功能 状态密切相关 (Zhu, 2014)。《灵枢・邪气脏腑病形》中写道: “十二经脉, 三百六十五络, 其血气皆上注于面而走空䛎”。 由此可见, 气血的运行也可影响皮肤的状态。

气血失调的常见为气虚、血瘀。其中, 脾气虚是较为常见的 气血不足症状。脾为 “后天之本” “气血生化之源”, 主运化 而化生气血, 脾胃功能健旺可以滋养人体正气, 是增强人体抗
病能力的基础。脾虚证大多由饮食不节、劳倦过度、忧思伤脾 或年老体衰、久病耗气所致(Zhang et al. , 2010)。

气血失调对皮肤产生的影响尚未见到有明确报道。本研究 通过建立脾气虚的小鼠模型, 评价脾气虚对小鼠皮肤指标的影 响。同时，在脾气虚小鼠模型皮肤使用多糖凝胶。拟将四个多 糖, 分别作用于脾气虚动物模型, 观察多糖因子对脾气虚小鼠皮 肤生理指标及皮肤切片组织的影响, 旨在确定多糖是否对脾气 虚皮肤有改善情况。

多糖是由 10 个以上单糖通过糖苷键连接而成的聚糖, 在自然 界分布广泛, 是组成高等植物、动物细胞膜及微生物细胞壁 的天然大分子物质田。多糖资源丰富、来源广泛, 可以从陆 地植物、海藻中提取, 也可以通过动物提取和微生物（细菌和 真菌) 发酵进行生物合成 (Qing et al., 2018)。现代药理学 研究表明, 多糖在免疫调节、抑制肿瘤、降血糖、降血脂、 
抗病毒、抗衰老、抗氧化等方面均有显著生物活性 (Chen et a1., 2016a)。相比具有如刺激性等安全风险的化学活性成分 而言, 中药来源的天然成分具有安全、温和、持久、高效等优 点, 成为现在功效化妆品研究开发的一大趋势 (Zhang et al., 2007)。中药多糖作为一种从传统中草药中提取的一类多糖化 合物, 大量的研究表明其具有延缓皮肤衰老、美白、晒后修 复、祛痘抗炎、促进伤口愈合等生物活性, 可以作为功效化妆 品活性成分的天然来源 (Lü et al., 2016)。

石斛, 甘, 微寒。归胃、肾经。具有益胃生津, 滋阴清热 的功效, 俗称 “千年润” 。现代药理表明石斛具有增强免疫 力、抗氧化、抗肿瘤、保肝、抗疲劳等多种功能活性 (Chen et al., 2016b)。其中石斛多糖是石斛的中药活性成分, 具有较强 的抗肿瘤、抗氧化和增强免疫功能活性 (An \& He, 2014)。陈 默等对精纯的石斛多糖提取物的保湿性能进行了评价, 表现出 较好的保湿性能, 并且有抗干燥损伤的防护作用, 可作为一种 天然植物类保湿剂应用于化妆品中 (Chen et al., 2015)。

天山雪莲, 是维吾尔族常用药, 具有补肾活血、强筋骨通 经络的功效。雪莲中的多糖对紫外线具有很强的吸收作用 (Guo et al., 2015)。

银耳, 是一种大型高等真菌, 属于有隔担子菌亚纲银耳科, 重要的药食同源物种。研究发现, 银耳多糖具有促进机体免疫 功能、抗衰、抗氧化的作用, 可作为保湿美白剂应用于化妆品 中(Huang et al., 2013)。

\section{Methods}

\section{1. 实验材料}

\section{1) 实验动物}

BLAB/c小鼠(Charles River, China), 此性, 体重19-21g, 60 只。

\section{2) 实验试剂}

本文所使用实验试剂如下: 薇婷脱毛亳 (depilatory cream, Veet, China)、猪脂、生理盐水、石斛多糖（Dendrobium polysaccharides, Foshan Allan Conney Biotechnology Co., Ltd., China)、雪莲多糖 (Saussurea ivolucrata polysaccharides, Foshan Allan Conney Biotechnology Co., Ltd. ) 、银耳多糖 (Tremella polysaccharides, Foshan Allan Conney Biotechnology Co., Ltd.)、去离子水 (deionized water, Aladdin, China)、丁二醇(butanediol, Aladdin, China)、EDTA二钠 (EDTA-Na2, Aladdin, China)、甘油 (glycerinum, Aladdin, China)、丙烯酸 (酯) 类/C10-30烷 醇丙烯酸酷交联聚合物 (acrylates/c10-30 alkanol acrylate crosslinked polymers, Aladdin, China)、氢氧化钠 $(\mathrm{NaOH}$, Aladdin, China)

\section{3) 仪器}

本文所使用的实验仪器如下：皮肤水分含量测试仪及测试探 头 (Corneometer ${ }^{\circledR}$ CM825; Courage+Khazaka electronic GmbH, Germany）、皮肤水分流失测试仪及测试探头（Tewamater® TM300, Courage+Khazaka electronic GmbH）、皮肤油脂分泌 测试及油脂测试盒 (SubumeterSM810) 、皮肤红黑色素测试仪 及测试探头 (Mexameter MX18; Courage+Khazaka electronic $\mathrm{GmbH}$ ) 、皮肤颜色测试仪及测试探头（Colorimeter， Courage+Khazaka electronic $\mathrm{GmbH}$ ) 、皮肤光泽度测试探头 (GL200; Courage+Khazaka electronic GmbH)。

\section{2. 实验方法}

\section{1) 多糖凝胶的制备}

(1) 在称重过的 $250 \mathrm{~mL}$ 烧杯中称取去离子水, 用称量纸称取 U20, 将其均匀撒在水中。静置约 $5 \mathrm{~min}$ (注意: 不要摚拌, 自 然分散润湿)，分散浸润至白色粉末完全润湿。

(2) 在浸润后体系中, 依次称取丁二醇、甘油, 摚拌加热升温 至 $80-85^{\circ} \mathrm{C}$, 摚拌 $5-10 \mathrm{~min}$, 转速 $35-40$ 转 $/ \mathrm{min}$, 摚拌均匀后降 温。体系中有絮状物属正常现象。

(3) 用 $50 \mathrm{~mL}$ 烧杯依次称取B相EDTA-2Na、离子水, 摚拌5-10 $\min$, 转速 $35-40$ 转 $/ \mathrm{min}$ 摚拌溶解至透明 (可适当加热, 加快溶 解, 温度 $55-60^{\circ} \mathrm{C}$ ) 待用。

(4) 分别取雪莲糖脂 $0.4 \mathrm{~g}$, 银耳多糖 $3 \mathrm{~g}$, 石斛多糖 $3 \mathrm{~g}$, 雪莲 糖脂、银耳多糖、石斛多糖各 $0.8 \mathrm{~g}$ 混合。用适量去离子水将多 糖溶解, 作为样品一、样品二、样品三和样品四。

(5) $\mathrm{A}$ 相降温至 $45^{\circ} \mathrm{C}$, 搅拌混合均匀后加入 $\mathrm{NaOH}(10 \%)$ 中和 (用量为 $\mathrm{U} 20$ 的 2.5 倍), (注意: 边搅拌边加, 不能过快), 加 入待用溶液B相和多糖样品, 摚拌形成透明啫喱。加入MTI摚拌 混合均匀。

（6）降至室温后称量, 装入已经灭菌的样品瓶。 样品一: $0.4 \%$ 雪莲糖脂凝胶, 样品二: $3 \%$ 银耳多糖凝胶, 样品 三: $3 \%$ 石斛多糖凝胶, 样品四: 0.8 等比混合多糖凝胶。制备完 成。详见Table 1。

\section{2) 分组、建模及给药}

(1) 动物分组

将健康小鼠饲养 4 天以适应环境后, 随机将小鼠分为 6 组, 每组 10只。

\section{(2) 脾气虚建模}

模型组: 隔日灌胃猪油 $20 \mathrm{~mL} / \mathrm{kg}$ 1次, 每日喂饲甘蓝 ( $\mathrm{Li}$, 2011)。 
对照组: 隔日灌胃生理盐水 $20 \mathrm{~mL} / \mathrm{kg}$ 1次, 每日喂饲料。

雪莲组: 隔日灌胃猪油 $20 \mathrm{~mL} / \mathrm{kg}$ 1次, 每日喂甘蓝。每日涂 抹样品 1 , 涂抹量 $2 \mathrm{mg} / \mathrm{cm}^{2}$ 。

银耳组: 隔日灌胃猪油 $20 \mathrm{~mL} / \mathrm{kg}$ 1次, 每日喂甘蓝。每日涂 抹样品 2 , 涂抹量 $2 \mathrm{mg} / \mathrm{cm}^{2}$ 。

石斛组: 隔日灌胃猪油 $20 \mathrm{~mL} / \mathrm{kg}$ 1次, 每日喂甘蓝。每日涂 抹样品 3 , 涂抹量 $2 \mathrm{mg} / \mathrm{cm}^{2}$ 。

混合组: 隔日灌胃猪油 $20 \mathrm{~mL} / \mathrm{kg}$ 1次, 每日喂甘蓝。每日涂 抹样品 4 , 涂抹量 $2 \mathrm{mg} / \mathrm{cm}^{2}$ 。

\section{3) 检测指标}

(1) 体重

在实验前称量各组小鼠的体重, 造模结束后再次称量各组小 鼠的体重, 用统计学方法进行分析比较。

\section{(2) 脾、胸腺指数}

造模结束后, 处死小鼠。分别用分析天平称取胸腺和脾脏的 质量。通过公式脾指数=小鼠脾重量/小鼠体重, 胸腺指数=小鼠 胸腺质量/小鼠体重。计算小鼠的脾、胸腺指数, 并用统计学方 法进行分析。

(3) 皮肤生理指标测定
将鼠尾根部以上长 $3 \mathrm{~cm}$ 、宽 $2 \mathrm{~cm}$ 长方形皮肤上被毛除去, 脱 毛。用脱毛亳除去小鼠背部被毛, 用清水清洗后棉球擦拭掉水 分。此步骤需在皮肤测定前一天进行, 第二天, 使用皮肤生物 量化仪器测定皮肤指标。小鼠每隔 3 天（第 $1 、 4 、 7$ 天）进行一 次皮肤生物指标测定，检测其皮肤变化。

\section{(4) 皮肤组织切片染色}

小鼠解剖后取背部皮肤组织，固定于 $4 \%$ 多聚甲醛溶液中， 48 $\mathrm{h}$ 后取出流水冲洗, 梯度乙醇脱水, 二甲苯透明, 浸蜡包埋, 切 片, HE染色, 中性树胶封片, 显微镜下观察小鼠皮肤组织有无 病理形态学改变, 进行组间比较和照相。

\section{3. 统计学方法}

采用统计软件SPSS ver. 25.0 software (SPSS Inc., USA) 版统计, 计量资料数值用均数土标准差 $(x \pm s)$ 表示。

\section{Results and Discussion}

\section{1.生物表征观察}

通过观察脾气虚组动物生物表征 (Zhou et al., 2001), 判断

Table 1. Gel preparation formula

\begin{tabular}{|c|c|c|c|}
\hline Phase & Raw material & Standard Chinese name & Added amount (wt\%) \\
\hline \multirow{4}{*}{ A } & U20 & Acrylate (C10-30) alkanol acrylate cross-linked polymer & 0.80 \\
\hline & Deionized water & Deionized water & To100 \\
\hline & Butanediol & Butanediol & 2.00 \\
\hline & Glycerin & Glycerin & 4.00 \\
\hline \multirow{3}{*}{ B } & EDTA-2Na & EDTA disodium salt & 0.05 \\
\hline & Deionized water & Deionized water & 10.00 \\
\hline & Polysaccharide & Polysaccharide sample & Add as above \\
\hline \multirow{2}{*}{ C } & $\mathrm{NaOH}(10 \%)$ & Sodium hydroxide & 1.80 \\
\hline & MTI & Methyl isothiazolinone/iodopropynol butyl carbamate & 0.15 \\
\hline
\end{tabular}

Table 2. Observation of spleen Qi deficiency type biological characterization

\begin{tabular}{ll}
\hline Observation item & Mental burnout, lying, lazy, arched back, grouped, eyes. In severe cases, \\
there is a lack of spirit when shocked, struggling when grasping, and hesitation & Dry, shrunken, and dull \\
Skin appearance & Coat is yellowish or even scarce. Ear tails are white \\
Coat color & Soft stools, loose stools, and diarrhea \\
Poop & Slow weight gain or weight loss \\
Body weight & The tongue is pale \\
Tongue & Body temperature does not change or decreases slightly \\
Body temperature & Reduced appetite \\
Other &
\end{tabular}


造模成功。脾气虚证型的生物表征如Table 2. 所示。

脾气虚模型组小鼠开始出现明显精神倦怠。啫卧, 懒动, 弓 背, 扎堆, 咪眼。有泄泻的现象, 肛门周围污秽。体重下降, 体温降低。具体生物表征观察对比结果见Table 3。对照组小鼠 毛色光泽饱满, 模型小鼠毛色枯槁稀疏。经统计软件分析, 与 对照组相比，模型组体重具有极显著性差异 $(p<0.01)$ 。造模时 间七天。

\section{2. 脾和胸腺指数}

脾气虚模型组小鼠胸腺指数和脾指数均高于对照组, 且呈显 著性差异，表明造模成功。脾指数、胸腺指数结果见Table 4 。 与对照组相比, $p<0.05,{ }^{*}$ 表示差异显著; $p<0.01$, ${ }^{* *}$ 表示差异极 显著。

\section{3. 皮肤测试指标}

按照脾气虚造模方式喂养第七天，进行皮肤生物指标测试。 皮肤指标的测定于每只小鼠背部同一部位, 水含、光泽、颜 色、红黑色素测定 5 次, 取平均值; 水散、油脂测一次。统计 发现, 模型组水分含量极显著降低, 光泽度显著降低, 油脂含 量极显著升高。多糖给药组皮肤指标相比于模型组, 皮肤水分 含量明显升高, 光泽度明显升高, 油脂含量极显著降低。测 试结果见Table 5。与模型组相比, $p<0.05$, “表示差异显著; $p<0.01,{ }^{* *}$ 表示差异极显著。

\section{4. 皮肤组织切片结果}

皮肤切片光镜下随机选取 5 个视野。其观察要点有以下几 点: (1)角质层, 是否角化过度或角化不全; (2)毛囊发育情况; (3)皮脂腺油脂分泌情况; (4)真皮层单一核及多核细胞浸润; (5)

Table 3. Observation results of spleen Qi deficiency type biological characterization

\begin{tabular}{lccccccccc}
\hline & $\begin{array}{l}\text { Signs and } \\
\text { symptoms }\end{array}$ & $\begin{array}{c}\text { Skin } \\
\text { appearance }\end{array}$ & Coat color & Poop & Body weight (g) & Tongue & $\begin{array}{c}\text { Body } \\
\text { temperature }\end{array}$ & Appetite \\
Model group & ++ & ++ & ++ & ++ & $16.35 \pm 0.83$ & ++ & ++ & ++ \\
Contorl group & - & - & - & - & $19.61 \pm 0.67$ & - & - & - \\
Saussurea involucrate group & + & + & + & + & $15.85 \pm 0.74$ & + & + & + \\
Tremella group & + & + & + & + & $17.20 \pm 0.83$ & + & + \\
Dendrobium group & + & + & + & + & $16.72 \pm 0.70$ & + & + \\
Mixed group & + & + & + & + & $16.56 \pm 0.60$ & + & + \\
\hline
\end{tabular}

Note: "+", indicates the phenomenon described in Table 3. The more "+", the more obvious the phenomenon. "-", indicates that the phenomenon described in Table 3 does not occur.

Table 4. Comparison of spleen and thymus indexes

\begin{tabular}{lcccccc}
\hline & Model group & Control group & $\begin{array}{c}\text { Saussurea } \\
\text { involucrate group }\end{array}$ & Tremella group & Dendrobium group & Mixed group \\
Spleen index (\%) & $0.0024 \pm 0.0006^{* *}$ & $0.0048 \pm 0.0008$ & $0.0032 \pm 0.0004^{*}$ & $0.0031 \pm 0.0008^{*}$ & $0.0033 \pm 0.0005^{*}$ & $0.0036 \pm 0.0004^{*}$ \\
Thymus index (\%) & $0.0012 \pm 0.0006^{* *}$ & $0.0026 \pm 0.0008$ & $0.0021 \pm 0.0011^{*}$ & $0.0011 \pm 0.0004^{*}$ & $0.0016 \pm 0.0003^{*}$ & $0.0017 \pm 0.0005^{*}$ \\
\hline
\end{tabular}
${ }^{*} p<0.05$.

\section{Table 5. Comparison of skin indicators}

\begin{tabular}{|c|c|c|c|c|c|c|}
\hline & Model group & Control group & $\begin{array}{c}\text { Saussurea } \\
\text { involucrate group }\end{array}$ & Tremella group & Dendrobium group & Mixed group \\
\hline Moisture content & $14.76 \pm 3.23$ & $41.00 \pm 3.44^{* *}$ & $32.16 \pm 7.93^{* *}$ & $27.32 \pm 3.65^{*}$ & $26.10 \pm 6.07^{*}$ & $27.80 \pm 5.15^{*}$ \\
\hline Percutaneous water loss & $14.64 \pm 4.90$ & $14.05 \pm 3.47$ & $14.46 \pm 2.23$ & $23.06 \pm 5.60$ & $21.23 \pm 6.05$ & $20.36 \pm 4.01$ \\
\hline Gloss & $1.49 \pm 0.32$ & $3.55 \pm 0.70^{* *}$ & $2.69 \pm 0.56^{*}$ & $2.27 \pm 0.67^{*}$ & $2.23 \pm 0.33^{*}$ & $2.71 \pm 0.26^{*}$ \\
\hline MI & $93.56 \pm 11.95$ & $82.42 \pm 15.49$ & $103.92 \pm 25.29$ & $90.08 \pm 12.68$ & $88.18 \pm 12.59$ & $82.76 \pm 19.22$ \\
\hline El & $280.24 \pm 32.83$ & $251.50 \pm 18.84$ & $313.32 \pm 43.89$ & $355.50 \pm 44.71$ & $324.94 \pm 35.86$ & $325.70 \pm 24.76$ \\
\hline L & $55.08 \pm 0.95$ & $58.65 \pm 1.79$ & $56.79 \pm 1.66$ & $55.91 \pm 1.42$ & $56.76 \pm 1.09$ & $56.28 \pm 0.71$ \\
\hline a & $5.93 \pm 1.46$ & $7.03 \pm 0.66^{*}$ & $7.02 \pm 1.14^{*}$ & $7.81 \pm 0.77^{*}$ & $8.10 \pm 0.60^{*}$ & $7.73 \pm 0.62^{*}$ \\
\hline$b$ & $-1.26 \pm 0.67$ & $-1.17 \pm 0.46$ & $-0.47 \pm 0.98$ & $-0.96 \pm 0.53$ & $-0.80 \pm 0.58$ & $-0.67 \pm 0.40$ \\
\hline ITA & $0.00 \pm 0.00$ & $0.00 \pm 0.00$ & $0.00 \pm 0.00$ & $0.00 \pm 0.00$ & $0.00 \pm 0.00$ & $0.00 \pm 0.00$ \\
\hline Grease & $22.11 \pm 26.12$ & $1.10 \pm 1.45^{* *}$ & $5.60 \pm 13.95^{* *}$ & $7.25 \pm 4.83^{* *}$ & $16.44 \pm 20.49^{*}$ & $14.00 \pm 15.35^{*}$ \\
\hline
\end{tabular}

MI, melanin; El, red pigment. ITA, individual typology angle; $p<0.05 ;{ }^{* *} p<0.01$. 
毛细血管扩张。

皮肤切片图片见Figure 1-6所示。模型组小鼠皮肤组织相较
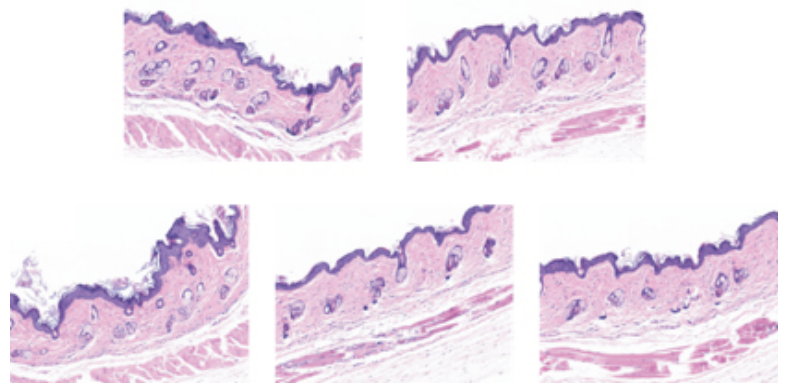

Figure 1. Model group skin tissue section.

Keratinocyte hyperplasia, hair follicle hypoplasia, more hair follicle oil, thicker dermis, eosinophil invasion, and less vascular endothelial cells.
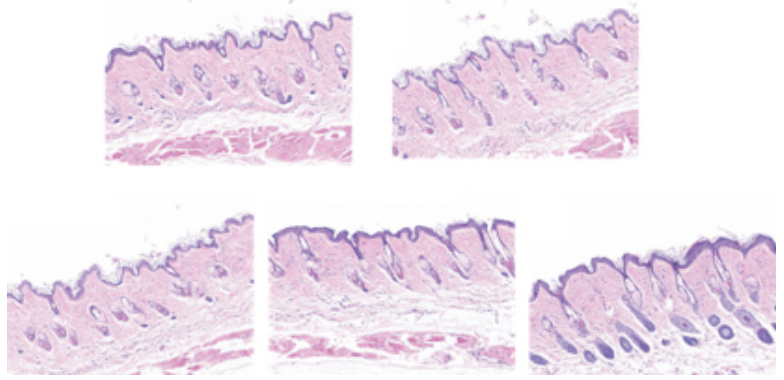

Figure 3. Skin tissue section of saussurea involucrate group.

Keratinocyte hyperplasia basically recovered, hair follicles developed more normally, hair follicles had less oil, and the dermis layer was slightly thickened. No infiltration of eosinophils and normal vascular endothelial cell density were observed.
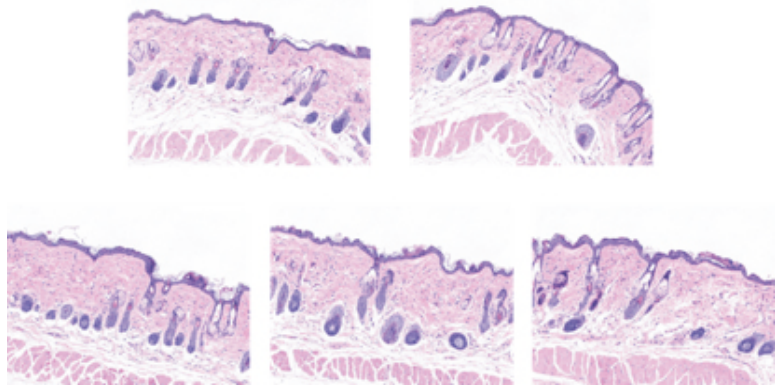

Figure 5. Skin tissue section from Dendrobium group.

Keratinocyte proliferation is heavier, the hair follicles are less developed, the hair follicles have more oil, the dermis is thicker, the borders are unclear, infiltration of eosinophils and multinucleated cells, and high density of vascular endothelial cells are seen.
于正常组存在角质细胞增生、毛囊发育不完全且油脂分泌较 多、血管内皮细胞较少的情况，印证了脾气虚对皮肤组织结构
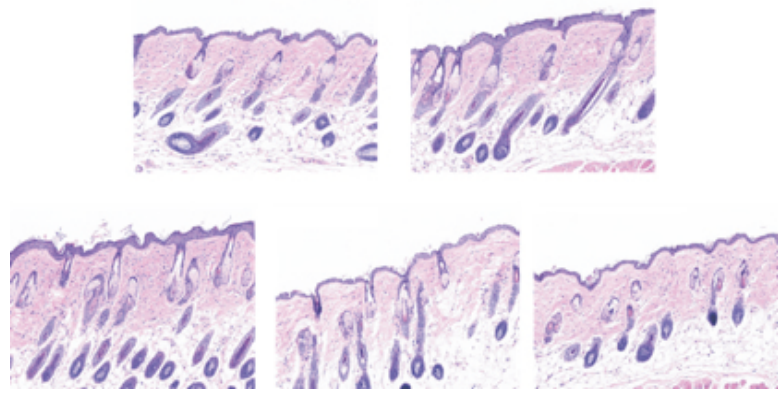

Figure 2. Normal group skin tissue section.

No keratinocyte proliferation, normal hair follicle development, less hair follicle fat, no thickening of the dermis, no infiltration of eosinophils, and normal distribution of vascular endothelial cells.
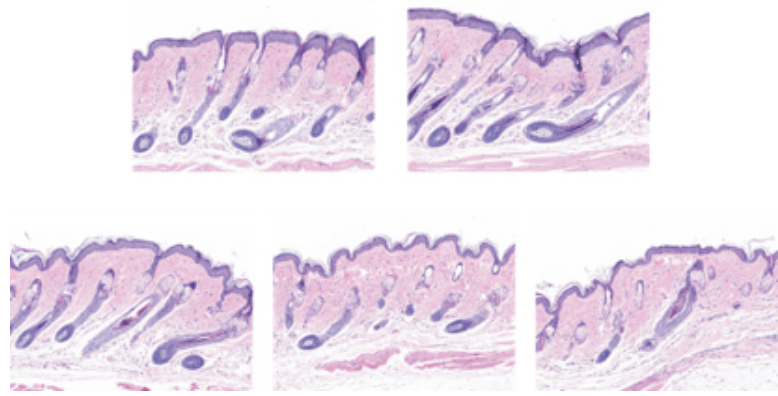

Figure 4. Skin tissue section of Tremella group.

The skin keratinocytes are proliferated, the hair follicles are normal, the hair follicles have less oil, and the dermis is thicker. A small amount of eosinophils and multinucleated cells infiltrate, and the density of vascular endothelial cells is close to normal.
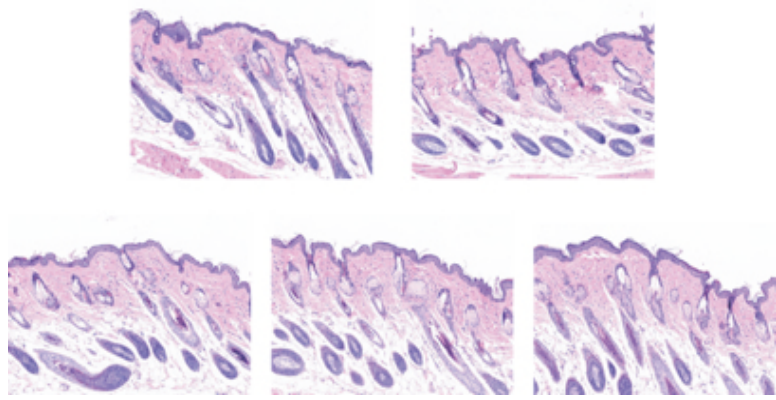

Figure 6. Mixed group skin tissue sections.

Keratinocyte hyperplasia is lighter, hair follicles are slightly more vigorously developed, hair follicles have less oil, and the dermis layer is close to normal. A small amount of multinucleated cells infiltrate and the density of vascular endothelial cells is slightly lower. 
存在不良影响。涂抹多糖凝胶的组别皮肤组织结构情况较模型 组有所改善, 但并不能完全达到正常组水平。给药组改善效 果: 雪莲组 >混合组 $>$ 银耳组 $>$ 石斛组。

\section{Conclusion}

\section{1. 模型评价}

脾虚模型建立成功的标准主要是通过表征观察、体重、皮肤 指标测定、脾和胸腺指标和对照组进行对比来判断。从表征观 察上看, 模型组的毛发明显变稀疏, 且神情倦急、便溏泄泻。 体重上, 模型组与对照组间的差异极显著 $(p<0.01)$ 。皮肤指 标的测定上, 模型组与对照组在水分含量、光泽度、油脂上具 有极显著差异 $(p<0.01)$ 。脾脏和胸腺, 模型组明显小于对照 组; 计算其脾指数和胸腺指数, 在统计学上, 具有极显著差异 $(p<0.01)$ 。

\section{2. 多糖凝胶改善脾气虚状态相关性}

涂抹多糖凝胶组小鼠从毛发上看, 仍比对照组稀疏, 同时会 出现便溏的现象。体重上, 给药组小鼠的体重明显低于对照组 具有极显著差异 $(p<0.01)$, 与模型组无明显差异。在皮肤指 标上, 水含、光泽度明显高于模型组, 但低于对照组, 两两间 差异极显著; 油脂低于模型组, 高于对照组, 且具有极显著差 异。脾指数和胸腺指数给药组都介于模型组与对照组之间, 且 具有极显著性差异 $(p<0.01)$ 。由皮肤组织切片的结构观察情 况, 表明在皮肤角质细胞、毛囊发育及分泌油脂情况, 多糖组 相比于模型组都有一定的改善。

\section{3. 四种多糖凝胶改善脾气虚状态的比较}

涂抹多糖凝胶主要对小鼠的皮肤指标有显著的改善作用。 混合多糖与雪莲糖脂对皮肤光泽度改善效果最为明显, 其次是 银耳, 石斛多糖次之。模型组油脂远高于正常组。雪莲多糖明 显改善因脾虚而导致的油脂分泌过多的现象。银耳多糖对油脂 分泌的改善情况也十分显著, 混合多糖次之, 石斛多糖效果最 弱, 但也具有明显的改善作用。脾气虚模型皮肤水含的改善情 况, 雪莲组最优, 其次是混合多糖组, 最后是银耳、石斛多 糖。

多糖凝胶对脾气虚模型的水含、光泽、油脂含量、皮肤组 织结构形态有明显的改善效果。但对脾虚小鼠的体重改善不明 显。故, 外用多糖凝胶虽对皮肤部分测试指标有所改善, 但对 脾气虚的体质以及肉眼可见的表征改变不明显。

四个多糖凝胶对脾气虚皮肤指标改善情况, 雪莲组〉混合组〉 银耳组 >石斛组。对皮肤指标改善的主要方面为皮肤的水含、油 脂和光泽度。

\section{Author's contribution}

BC designed, performed experiments, analyzed data, and wrote the manuscript. JRL designed all experimental investigations, and developed the process. LNL performed histology experiments and collected data. LNL, JRL and LL performed mouse experiments. HM and JL oversaw the study. All figures are created by the author and the coauthors.

\section{Author details}

Bill Che (Director), Department of Research and Development Daily Chemicals Cosmetology, Infinitus Co. Ltd., 17th floor, Infinity center, 12 Zhujiang west road, Zhujiang new town, Tianhe district, Guangzhou 510000, China; Linan Li (Engineer), Department of Research and Development Daily Chemicals Cosmetology, Infinitus Co. Ltd., 17th floor, Infinity center, 12 Zhujiang west road, Zhujiang new town, Tianhe district, Guangzhou 510000, China; Jiarui Li (Graduate student), Department of Cosmetology, Beijing Technology and Business University, 11 Fucheng Road, Haidian District, Beijing 100048, China; Li Li (Professor), Department of Cosmetics, Beijing Technology and Business University, 11 Fucheng Road, Haidian District, Beijing 100048, China; Hong Meng (Professor), Department of Cosmetics, Beijing Technology and Business University, 11 Fucheng Road, Haidian District, Beijing, 100048, China; Jim Liu (Manager), Department of Research and Development Daily Chemicals Cosmetology, Infinitus Co. Ltd., 17th floor, Infinity center, 12 Zhujiang west road, Zhujiang new town, Tianhe district, Guangzhou 510000, China.

\section{References}

An FJ, He YX. Research advance of polysaccharide from Dendrobium nobile Lindl. Journal of Anhui Agricultural Sciences, 42: 3857-3562, 2014.

Chen M, Sun Y, Zhao Y. Study on moisturizing effect of Dendrobium officinale. Acta Universitatis Traditionis Medicalis Sinensis Pharmacologiaeque Shanghai, 29: 70-73, 2015.

Chen GM, Wang SC, Wang L, Du P, Shen HY. A review on treating skin photoaging with polysaccharide of Chinese 
medicine. Journal of Nanjing University of Traditional Chinese Medicine, 32: 396-400, 2016.

Chen GM, Wang SC, Wang L, Pei D, Shen HY. A review on treating skin photoaging with polysaccharide of Chinese medicine. Journal of Nanjing University of Traditional Chinese Medicine, 32: 396-400, 2016.

Guo Y, Sun J, Wang LW. Alleviation of inflammatory damage induced by the apoptosis of HaCaT cells after ultraviolet B radiation by Saussurea tridactyla Sch-Bip. polysaccharides through P38 MAPK passage. Chinese General Practice, 18: 3314-3319, 2015.

Huang YJ, Jia FX, Shi WJ. Research progress of Tremella polysaccharides. Chongqing Chinese Herbal Medicine Research, 1: 43-47, 2013.

Li YM, Han YM, Zhu KJ. Comprehensive evaluation of symptoms of deficiency in spleen deficiency model mice and study of defects. Hunan Journal of Traditional Chinese Medicine, 27: 126-127, 2011.

Lü CP, Xiong Z, He QQ, Yeon J. Application prospects of polysaccharide from traditional Chinese medicine used in functional cosmetics. Flavour Fragrance Cosmetics, 3: 67-71, 2016.

Qing S, Yu SR, Bai Y, Yao Q, Wang P. Research progress on immunological mechanism of spleen deficiency syndrome. Journal of Jiangxi University of Traditional Chinese Medicine, 30: 116-120, 2018.

Qiu T. Study on enlightenment and application of thinking of inner canon of Huangdi on modern Chinese medicine. Smart Healthcare, 3: 184-185, 2018.

Zhang $\mathrm{H}$, Wang ZY, Wang X, Yao L, Wu Zg, Yang X. Research advancements of chemical modification of polysaccharide and their biological activities. Food and Fermentation Industries, 36: 102-107, 2010.

Zhang M, Chen SL. Review of current situation and prospects of research and development on traditional Chinese medicine cosmetics. China Journal of Chinese Materia Medica, 32: 2457-2460, 2007.

Zhu H. Study of cosmetology theory in treatise on febrile and miscellaneous disease. Chinese Journal of Traditional Chinese Medicine and Pharmacy, 29: 3064-3066, 2014.

Zhou S, Zhou Y, Fan Y, Zhang Y, Jiang X, Cui C, Wang S, Chen X. Preliminary study on standardization of animal model of spleen Qi deficiency-syndrome: partition in macroscopic syndrome and symptoms. Chinese Journal of Integrated Traditional and Western Medicine on Digestion, 9: 264267, 2001. 


\section{中文摘要}

\section{多糖凝胶对脾气虚症状皮肤改善情况的相关性研究}

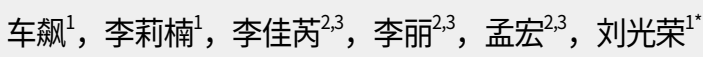

无限极（中国）有限公司日化产品研发部，广州, 中国

${ }^{2}$ 北京工商大学化妆品系, 北京, 中国

3北京市植物资源研究开发重点实验室，北京，中国

目的: 验证多糖凝胶对脾气虚, 尤其是脾气虚所导致的皮肤现象有改善作用。方法: 用饮食失节的方法建立脾气 虚证的小鼠模型，用制备四种多糖凝胶涂抹在小鼠背部皮肤，用体重、脾胸腺指数、皮肤切片、皮肤指标等进 行评价。结果: 脾气虚证小鼠含水量较低, 光泽度较低, 油脂分泌增加, 与对照组比较差异有统计学意义 $(p$ $<0.01 ） 。$ 多糖凝胶作用于脾气虚的小鼠, 与模型组相比, 皮肤水分增加, 光泽增加, 油脂分泌减少, 具有显着 意义。结论: 多糖凝胶具有改善脾气虚症状, 尤其是脾气虚所导致的皮肤干燥无光泽的现象, 为后续多糖在化妆 品改善脾气虚的皮肤症状上的应用提供依据。

关键词: 脾气虚, 皮肤, 石斛多糖, 银耳多糖, 雪莲多糖 


\section{국문초록}

\section{다당류젤과 비장 기 결핍 증상의 상관 관계}

차표 ${ }^{1}$, 리려남 ${ }^{1}$, 리가열 ${ }^{2,3}$, 리려, ${ }^{2,3}$ 맹홍 2,3 , 류광영 ${ }^{*}$

${ }^{1}$ 무한급(중국)유한회사일화제품연구부, 광주, 중국

${ }^{2}$ 북경공상대학 화장품학과, 북경, 중국

${ }^{3}$ 북경시식물자원연구개발중점연구실, 북경, 중국

목적: 본 연구는 다당류젤이 비장 기 결핍을 완화하거나 또는 비장 기 결핍으로 인한 피부 현상을 개선 할 수 있는 지 확인하기 위한 것이다. 방법: 비장 기 결핍 증후군의 마우스 모델은 섭식 장애에 의해 확립되었다. 마우스 등 피부에 4 종의 다당류젤을 도포하고, 무게, 비장-흥선 지수, 피부 섹션 및 피부 지수를 평가하였다. 결과: 비장 기 결핍 생쥐는 물 함량이 낮고, 피부 광택이 적으며, 기름 분비가 증가했다. 이는 대조군과 유의한 차이가 있었다( $p<0.01)$. 다당류젤을 비장 기 결핍이 있는 마우스에 도포 시, 모델 그룹과 비 교하여 피부 수분 함량 증가, 광택 증가, 오일 분비가 감소하는 효과를 나타냈다. 결론: 다당류젤은 비장 기 결핍의 증상, 특히 비장 기 결핍으로 인해 건조하고 칙칙한 피부를 개선하는 효과를 나타냈으며, 이후 비장 기 결핍의 피부 증상을 개선하기 위해 화장품에 다당류젤을 적용할 수 있는 기초적인 자료를 제공함에 의의가 있다고 사료된다.

핵심어: 비장 기 결핍, 피부, 석곡다당, 은이다당, 설련다당 
